\title{
Aktiv in jedem Alter - Sport und Ernährung in den verschiedenen Lebensphasen: Kinder
}

\author{
Active at Any Age - Exercise and Nutrition in Different Phases of Life: Children
}

Autor

Institut
C. Graf

Institut für Bewegungs- und Neurowissenschaft, Deutsche Sporthochschule Köln, Abteilung für Bewegungs- und Gesundheitsförderung

\section{Schlüsselwörter \\ - Bewegungsmangel \\ - Gesundheit \\ - Adipositas \\ - Bildschirmzeit \\ - Prävention \\ - Familie \\ - Politik}

Keywords

- lack of exercise

$\checkmark$ health

- obesity

- screen time

- prevention

- family

- politics

\section{Zusammenfassung}

\section{$\nabla$}

Bewegung ist für eine gesunde, ganzheitliche Entwicklung von Kindern essenziell. Sie verbessert die körperliche wie kognitive Leistungsfähigkeit, schützt vor Zivilisationskrankheiten, Sucht bzw. Unfällen und steigert das Selbstwertgefühl, allerdings bietet die Lebenswelt von heute Kindern nicht genügend Bewegungsmöglichkeiten. Anstelle dessen beherrscht die Nutzung von audiovisuellen bzw. Bildschirm-Medien zunehmend die Freizeit von Kindern und Jugendlichen. Die Folgen sind vor allem Übergewicht und Adipositas sowie motorische Defizite, aber auch eine Abnahme der akademischen Leistung und eine Zunahme von Verhaltensauffälligkeiten.

Verstärkte Gesundheitsförderung und effektivere Präventivmaßnahmen sind notwendig und sollten in Schulen, Vereinen, Kindergärten und Kommunen stattfinden. Politik und Wirtschaft sind aufgefordert, die notwendigen Rahmenbedingungen zu schaffen.

Gesunde Kinder brauchen Bewegung. Das zeigen die Ergebnisse aller relevanten Studien: Sie beugt nicht nur kardiovaskulären Risikofaktoren vor, sondern verbessert auch die motorische, körperliche und kognitive Leistungsfähigkeit. Untersuchungen mit Erstklässlern belegen, dass Konzentration und Koordination eng zusammenhängen: Kinder, die in puncto Koordination gut abschnitten, hatten auch eine gute Konzentration - und damit auch Vorteile in der Schule. Darüber hinaus ist Bewegung auch Suchtprävention. Studien aus den USA ergaben zum Beispiel, dass sportliche Jugendliche, die in Vereinen aktiv sind, weniger Marihuana rauchten. In Deutschland gibt es jedoch das Phänomen, dass im Verein aktive Kinder mehr Kontakt zu Alkohol haben. In Vereins- oder Sportheimen sollte Bierwerbung daher generell verboten werden und Rauchverbot gelten. Auch

\section{Abstract \\ $\nabla$}

Exercise is essential in order to ensure the healthy, holistic development of children. It improves physical and cognitive performance; protects against lifestyle diseases, addiction disorders, and accidents; and it increases selfconfidence. Today's living environments do not provide children with enough opportunities for exercise. Instead, the use of audio visual and screenbased media increasingly dominates children's and adolescents' leisure time. The consequences are mainly overweight and obesity as well as motor deficits, but also a decrease in academic performance and an increase in behavioural abnormalities.

Increased health promotion and effective preventive measurements are needed and should take place in schools, clubs and societies, nurseries, and communities. Politicians and economists are invited to create the necessary frame work conditions.

der Konsum von alkoholfreien Biergetränken sollte für Jugendliche tabu sein, da sie dadurch früh an den Geschmack alkoholischer Getränke gewöhnt werden.

Bewegung trägt außerdem zur Unfallprävention bei: Kinder mit guter Koordination können besser abrollen und sich bei einem Sturz besser abfangen. Und eine trainierte Auge-Fuß-Koordination ist für Sportarten wie Tennis oder Weitsprung zwar für die Ergebnisse wichtig, im Straßenverkehr aber essenziell. Diese Fähigkeiten lernen Kinder nicht am Bildschirm, sondern nur im echten Leben. Letztlich stärkt Bewegung auch das Selbstbewusstsein. Kein Medium kann Kindern so viele Kompetenzen vermitteln: Im Team spielen, auf einen Baum und wieder herunterklettern, diese Fähigkeiten lernt man nur im Sport $(\bullet$ Abb. 1) [1]. 


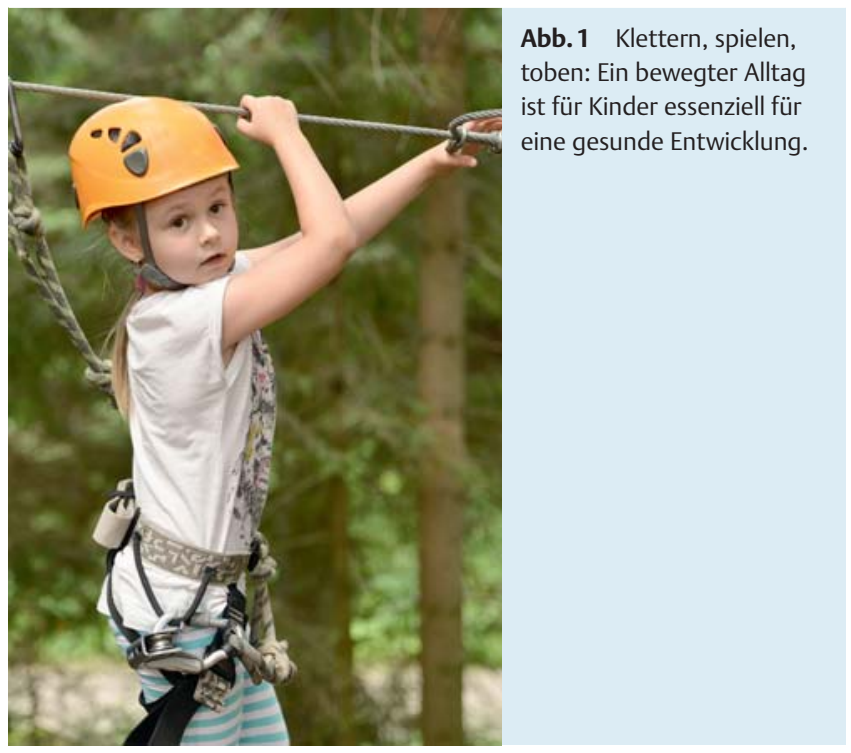

\section{Mehr Bewegung für kleine Kinder!}

$\nabla$

Kleine Kinder sollen sich so viel wie möglich bewegen, empfiehlt die National Association für Sport und Physical Education (NASPE) in ihrem letzten Update 2011 [2]. Dessen sollten sich alle Personen bewusst sein, die mit Kindern zu tun haben: Tagesmütter, Lehrpersonen, Erzieherinnen und vor allem die Eltern. Der Alltag vieler Kinder ist von Anfang an bewegungseinschränkend - sie sitzen erst im MaxiCosy, später fahren die Eltern sie überwiegend mit dem Auto. Früher war es normal, dass Kinder draußen spielten, heute sitzen sie vor dem Computer. Das natürliche Gespür dafür, wie viel Bewegung und wie viel Ruhe ein Kind braucht, ist in vielen Familien verloren gegangen.

Einer Metaanalyse zufolge bewegen sich Kinder heute sportlich nicht viel weniger als 1999 [3]. Eine differenzierte Betrachtung der Daten zeigt aber, dass die Bewegung pro Jahr im Alltag im Mittel um 7\% abgenommen hat [4]. In Deutschland kommen 27\% der Mädchen und Jungen auf die von der Weltgesundheitsorganisation empfohlenen 60 min körperliche Aktivität pro Tag [5]. Trotzdem nimmt die Fitness ab: Im Schnitt sinkt die Ausdauerleistungsfähigkeit alle 2 Jahre um $1 \%$. Ein fataler Trend, da sie einer der wichtigsten Prädiktoren für kardiovaskuläre und Tumorgesundheit sowie für die Vorbeugung von Demenz ist [6-8].

Jugenduntersuchungen in den USA und Deutschland zufolge ist die Fernsehzeit von 2002-2010 zwar leicht gesunken, konkret um $12-18$ min $[9,10]$. Im gleichen Zeitraum ist die Computerzeit während der Woche um 54, am Wochenende um 70 min gestiegen. Im Schnitt sitzen Jugendliche damit am Wochenende pro Tag 3 Stunden vor dem Fernseher plus zwei Stunden vor dem Computer, das macht rund 5 Stunden Bildschirmzeit pro Tag. Die Folgen: Weltweit ist ein Anstieg von Übergewicht und Adipositas zu beobachten - bei Erwachsenen um 27,5\%, bei Kindern um 47,1\%. Das entspricht einer Steigerung von 857 Millionen Adipösen und Übergewichtigen im Jahr 1980 auf 2,1 Milliarden im Jahr 2013. Zu diesem Ergebnis kommt eine Datenanalyse aus 188 Ländern aus 1769 Studien mit über 19000 Personen [11]. 2011 kam ein Review zu dem Ergebnis, dass die Prävalenz der Adipositas im Kindesalter ein Plateau erreicht habe. Dies ergaben die Daten von ca. 500000 Kindern und Jugendlichen aus 9 Ländern. Differenziert betrachtet ergeben sich jedoch für die einzelnen Länder große Unterschiede. In den Niederlanden stagniert die Prävalenz der kindlichen Adipositas seit Jahrzehnten [12], nicht aber bei türkischen oder marokkanischen Kindern. Dabei spielt der Sozialstatus eine große Rolle: Während in Gymnasien der Anteil übergewichtiger und adipöser Kinder bei 11\% liegt, beträgt er in Hauptschulen über 40\% [13].

\section{Forderung nach verbesserter Prävention \\ $\nabla$}

Die heutige Lebenswelt bietet Kindern nicht mehr genügend Bewegungsmöglichkeiten und befeuert die Entwicklung von Übergewicht und Adipositas. Deshalb ist der Ruf nach Prävention notwendig. Er beinhaltet 3 Kernforderungen:

1. Bessere Empfehlungen: 2014 einigten sich Experten von 20 Institutionen in einem Konsensus-Statement, Kindern und Jugendlichen nicht mehr nur 60 min Bewegung pro Tag zu empfehlen, sondern mindestens 90 min.

2. Besseres Verständnis von Prävention: Übergewicht und Bewegungsmangel unterliegen komplexen Einflussfaktoren, die eng vernetzt sind - angefangen von der Situation im Mutterleib über den Lebensstil, das Lebensmittelangebot, psychische Faktoren, gesellschaftliche Veränderungen bis hin zu politischen Rahmenbedingungen.

3. Bessere Vernetzung und politischer Rahmen: Prävention sollte konzertiert und in allen Bereichen stattfinden. Je kleiner das Kind, desto größer ist der Einfluss der Familie. Schule und Freundeskreis, soziale Gruppen und kulturelle Traditionen sind ebenfalls einflussreiche Faktoren. Städteplanung und kommunale Gesundheitsförderung, Organisations- und Wirtschaftsstrukturen sollten geeignete Rahmenbedingungen schaffen. Wichtig ist außerdem eine präventionsorientierte Politik und Gesetzgebung in Europa, die zum Beispiel eine Ampelkennzeichnung einführt und Eltern eine gesunde Lebensmittelauswahl erleichtert [14, 15].

\section{Interessenkonflikt \\ $\nabla$}

Die Autorin hat keinen Interessenkonflikt.

\section{Literatur}

1 Graf C, Beneke R, Bloch $W$ et al. Recommendations for Promoting Physical Activity for Children and Adolescents in Germany. A Consensus Statement. Obesity facts 2014; 7: 178-190

2 National Association for Sport and Physical Education. Position Statement 2011. http://www.shapeamerica.org/advocacy/positionstate ments/pe/loader.cfm?csModule=security/getfile\&pageid=4650 (Zugriff am 27.11.2015)

3 Li S, Treuth MS, Wang Y. How active are American adolescents and have they become less active? Obesity Reviews 2010; 11: 847ff

4 Dumith SC, Gigante DP, Domingues MR et al. Physical activity change during adolescence: a systematic review and a pooled analysis. International Journal Of Epidemiology 2011; 40: 685-698

5 Robert Koch-Institut. GBE Kompakt. Gesund aufwachsen - welche Bedeutung kommt dem sozialen Status zu? 2015: http://www.rki.de/DE/ Content/Gesundheitsmonitoring/Gesundheitsberichterstattung/GBE DownloadsK/2015_1_gesund_aufwachsen.html?nn=5945060 (Zugriff am 27.11.2015)

6 Tomkinson GR, Léger LA, Olds TS et al. Secular trends in the performance of children and adolescents (1980-2000): an analysis of 55 studies of the $20 \mathrm{~m}$ shuttle run test in 11 countries. Sports Medicine 2003; 33: $285-300$

7 Tomkinson GR. Global changes in anaerobic fitness test performance of children and adolescents (1958-2003). Scandinavian Journal Of Medicine \& Science In Sports 2007; 17: 497-507 
8 Dyrstad SM, Berg T, Tjelta LI. Secular trends in aerobic fitness performance in a cohort of Norwegian adolescents. Scandinavian Journal of Medicine \& Science In Sports 2012; 22: 822 - 827

9 Iannotti RJ, Wang J. Trends in physical activity, sedentary behaviour, diet, and BMI among US adolescents. Pediatrics 2013; 132: 606-614

10 Bucksch J, Inchley J, Hamrik Z et al. HBSC Study Group Germany: Trends in television time, non-gaming PC use and moderate-to-vigorous physical activity among German adolescents 2002-2010. BMC Public Health 2014; 14: 351ff

$11 \mathrm{Ng} \mathrm{M}$, Fleming T, Robinson M et al. Global, regional and national prevalence of overweight and obesity in children and adults 1980-2013: A systematic analysis. Lancet 2014; 6: $342-360$
12 Olds T, Maher C, Zumin S et al. Evidence that the prevalence of childhood overweight ist plateauing: data from nine countries. International Journal Of Pediatric Obesity 2011; 6: $342-360$

13 Graf C, Jouck S, Koch B et al. Motorische Leistungsfähigkeit von übergewichtigen und adipösen Kindern und Jugendlichen im Vergleich zu ihren Altersgenossen. Monatsschrift Kinderheilkunde 2007; 155: $631-637$

14 Lobstein T, Baur L, Uauy R. IASO: Obestiy in children and young people: a crisis in public health. Obesity Reviews 2004; 5: 4-85

15 Mehtälä MAK, Sääkslahti AK, Inkinen ME et al. A socio-ecological approach to physical activity interventions in childcare: a systematic review. International Journal of Behavioral Nutrition and Physical Activity 2014; 11: $22 \mathrm{ff}$ 